\title{
Increasing Optical Efficiency in the Telecommunication Bands of Strain-Engineered Ga(As, Bi) Alloys
}

\author{
E. Tisbi, ${ }^{1}$ E. Placidi $\odot,{ }^{2,3, \dagger}$ R. Magri, ${ }^{4}$ P. Prosposito,${ }^{5}$ R. Francini $\odot,{ }^{5}$ A. Zaganelli, ${ }^{1}$ S. Cecchi, ${ }^{6}$ \\ E. Zallo® ${ }^{6}$ R. Calarco, ${ }^{6,7}$ E. Luna, ${ }^{6}$ J. Honolka, ${ }^{8}$ M. Vondráček, ${ }^{8}$ S. Colonna, ${ }^{3}$ and F. Arciprete ${ }^{1,3, *}$ \\ ${ }^{1}$ Dipartimento di Fisica, Università di Roma "Tor Vergata”, Via della Ricerca Scientifica 1, I-00133 Roma, Italy \\ ${ }^{2}$ Department of Physics, Sapienza University of Rome, P.le Aldo Moro 2, 00185 Rome, Italy \\ ${ }^{3}$ Istituto di Struttura della Materia-CNR (ISM-CNR), Via del Fosso del Cavaliere 100, I-00133 Roma, Italy \\ ${ }^{4}$ Dipartimento di Scienze Fisiche, Informatiche e Matematiche (FIM), Università di Modena e Reggio Emilia, and \\ Centro S3 CNR-Istituto Nanoscienze, Via Campi 213/A, 41100 Modena, Italy \\ ${ }^{5}$ Dipartimento di Ingegneria Industriale, Università di Roma "Tor Vergata”, Via del Politecnico, I-00133 Roma, \\ Italy \\ ${ }^{6}$ Paul-Drude-Institut für Festkörperelektronik, Hausvogteiplatz 5-7, 10117 Berlin, Germany \\ ${ }^{7}$ CNR-IMM, Via Fosso del Cavaliere 100, I-00133 Roma, Italy \\ ${ }^{8}$ Institute of Physics, Academy of Sciences of the Czech Republic, Na Slovance 2, CZ-182 21 Praha 8, Czech \\ Republic
}

(Received 18 March 2020; revised 22 May 2020; accepted 22 May 2020; published 10 July 2020)

\begin{abstract}
The search for semiconducting materials with improved optical properties relies on the possibility to manipulate the semiconductors band structure by using quantum confinement, strain effects, and by the addition of diluted amounts of impurity elements such as Bi. In this study, we explore the possibility to engineer the structural and physical properties of the $\mathrm{Ga}(\mathrm{As}, \mathrm{Bi})$ alloy by employing different stress conditions in its epitaxial growth. Films with variable concentration of Bi are grown by molecular beam epitaxy on bare $\mathrm{GaAs}(001)$ crystals and on partially relaxed (In, Ga)As double buffer layers acting as stressors aiming to control the $\mathrm{Bi}$ incorporation into the alloy and improving the optical properties in terms of efficiency. A combination of several structural and electronic characterization techniques and dedicated density-functional-theory calculations allows us a systematic comparison between the samples grown under compressive and tensile strain. We demonstrate the possibility to grow $\mathrm{Ga}(\mathrm{As}, \mathrm{Bi}) \mathrm{under}$ different strain conditions without affecting its crystal quality. The different strain conditions strongly impact the $\mathrm{Bi}$ incorporation in the GaAs matrix and the luminescence properties of the sample. We find (i) a striking improvement of the photoluminescence with a strongly increased radiative efficiency when $\mathrm{Ga}(\mathrm{As}, \mathrm{Bi})$ is grown under tensile strain and (ii) an interesting higher redshift with respect to $\mathrm{Ga}(\mathrm{As}, \mathrm{Bi}$ ) grown compressively on GaAs. These two effects allow us to reach the important photoluminescence emission at $1.3 \mu \mathrm{m}$ with a $\mathrm{Bi}$ concentration as low as $4.9 \%$ compared to $7.5 \%$ needed for samples grown directly on GaAs. This is a significant achievement for the application of the $\mathrm{Ga}(\mathrm{As}, \mathrm{Bi})$ material in optoelectronic devices.
\end{abstract}

DOI: 10.1103/PhysRevApplied.14.014028

\section{INTRODUCTION}

Optics and photonics technologies are responsible for the development of devices that impact telecommunications, information processing and storage, as well as other important applications. Research on the optical properties of materials aims at using photons as information carriers, which are more efficient than electrons in the transport of information, and at reaching higher performances in terms

\footnotetext{
*fabrizio.arciprete@roma2.infn.it

†ernesto.placidi@uniroma1.it
}

of power consumption, efficiency, and speed, as demonstrated by the great impulse received by communication technology after the invention of optical fibers. The band structure of semiconductors can be manipulated by using quantum confinement, strain effects, and by the addition of small amounts of impurity elements. In this regard, the development of III-V semiconductors with dilute bismuth has been a topic of great interest among researchers in recent years $[1,2]$. The $\mathrm{Ga}(\mathrm{As}, \mathrm{Bi})$ alloy exhibits a bandgap reduction of up to $90 \mathrm{meV} / \% \mathrm{Bi}$, a strong enhancement of spin-orbit splitting (useful to suppress Auger recombination losses) and an almost temperature-insensitive band 
gap, [1,3-6] all of which are attractive properties for nearto mid-infrared lasers, spintronic devices, photodetectors, and terahertz optoelectronic applications. $\mathrm{Ga}(\mathrm{As}, \mathrm{Bi})$ light emission, for $\mathrm{Bi}$ contents ranging from 6 to $11 \%$, would cover the most important telecommunication bands (1.3 to $1.55 \mu \mathrm{m}$ ). However, the fabrication of a good-quality emitting material at these $\mathrm{Bi}$ concentrations represents a formidable and not yet accomplished task. [6] Indeed, the implementation of $\mathrm{Ga}(\mathrm{As}, \mathrm{Bi})$ is still limited due to the difficulty of growing a high-quality material with wellcontrolled properties. The scarce miscibility of $\mathrm{Bi}$ in $\mathrm{GaAs}$ and the strong tendency of Bi to segregate and form metallic clusters [7] require the adoption of nonstandard growth conditions that lead to the simultaneous incorporation of defects, which negatively affect the optical properties. The most used approach reported in the literature to obtain an efficient $\mathrm{Bi}$ incorporation during epitaxial growth of $\mathrm{Ga}(\mathrm{As}, \mathrm{Bi})$ is to act on the kinetic parameters of the growth, such as flux ratios, growth rate, and temperature $[6,7]$. In particular, low-temperature growth and 1:1 Asto-Ga flux ratio are required to favor Bi incorporation. The necessity to precisely control these parameters makes molecular beam epitaxy (MBE) the preferred technique for the growth of $\mathrm{Ga}(\mathrm{As}, \mathrm{Bi})$. Anyway, in the last decade the progress and/or development of alternative techniques or approaches for the growth of $\mathrm{Ga}(\mathrm{As}, \mathrm{Bi}$ ) was not as successful as desired [6]. As a matter of fact, the compressive strain induced by the growth of the alloy on a GaAs substrate also influences, increasingly with the $\mathrm{Bi}$ amount, the incorporation of $\mathrm{Bi}$ and the physical properties of the $\mathrm{Ga}(\mathrm{As}, \mathrm{Bi})$ film. The epitaxial growth of semiconductor thin films and heterostructures have often been manipulated by strain to tailor their structural and physical properties, allowing the realization of artificial structures not occurring in nature [8]. Based on these considerations, in the present paper we report on a growth approach aimed at strain engineering the pseudomorphic growth by MBE of $\mathrm{Ga}(\mathrm{As}, \mathrm{Bi})$ on $\mathrm{GaAs}(001)$. While a few papers have considered the effects of strain in $\mathrm{Ga}(\mathrm{As}, \mathrm{Bi})$ alloys, a real strain-engineering approach aiming to foster the $\mathrm{Bi}$ incorporation and improve optical properties in terms of efficiency, is still lacking. To this end, we grew suitable intermediate (In, Ga)As buffer layers acting either as compliant virtual substrates or as internal stressors to tune the strain conditions of the $\mathrm{Ga}(\mathrm{As}, \mathrm{Bi})$ layer. This approach enables us to grow $\mathrm{Ga}(\mathrm{As}, \mathrm{Bi})$ layers with similar $\mathrm{Bi}$ contents but different strain conditions, varying from compressive to tensile, as well as to grow $\mathrm{Ga}(\mathrm{As}, \mathrm{Bi})$ films under negligible strain for $\mathrm{Bi}$ concentrations as high as $7.2 \%$. We find a striking improvement of photoluminescence, with a strongly increased radiative efficiency when $\mathrm{Ga}(\mathrm{As}, \mathrm{Bi})$ is grown under tensile strain. This result demonstrates the large potential of strain-engineering concepts for tailoring the optical emissions of this material system. These results could impact telecommunication technology, e.g., optical fibers are widely used to transmit information by light carrier for long distances, and usually need optical amplifiers to regenerate the light pulses after attenuation. More efficient light sources reduce this need with consequent reduction of power consumption [9].

\section{EXPERIMENTAL AND THEORETICAL METHODS}

\section{A. Sample preparation}

Samples are grown by MBE using a solid-source MBE 32P Riber equipped with a conventional Knudsen effusion cell for As 4 . The substrate temperature is measured by an optical pyrometer with a nominal accuracy of $\pm 1{ }^{\circ} \mathrm{C}$. All $\mathrm{Ga}(\mathrm{As}, \mathrm{Bi})$ films are grown at the same substrate temperature $T=325^{\circ} \mathrm{C}$, with a quasistoichiometric As-to-Ga flux ratio, and a growth rate $0.5 \mathrm{ML} / \mathrm{s}$ for a thickness ranging from 60 to $170 \mathrm{~nm}$. To vary the Bi content four different $\mathrm{Bi}$ fluxes are used with a beam equivalent pressure in the range $1-8 \times 10^{-8}$ Torr. Semi-insulating $\operatorname{GaAs}(001)$ epiready substrates are used for all the samples. After standard cleaning and oxide removal procedures, an epitaxial GaAs buffer layer, $340 \mathrm{~nm}$ thick, is grown in As overflow at $590{ }^{\circ} \mathrm{C} . \mathrm{GaAs}_{1-x} \mathrm{Bi}_{x}$ films $(x=0.8 \%-7.5 \%)$ under compressive strain are grown on the GaAs buffer layer $(\mathrm{C} 1-\mathrm{C} 4$ in Table I). $\mathrm{GaAs}_{1-x} \mathrm{Bi}_{x}$ films $(x=0.7 \%-7.2 \%)$ under tensile strain (T1-T5, in Table I) are grown on top of a partially relaxed $\mathrm{In}_{y} \mathrm{Ga}_{1-y} \mathrm{As} / \mathrm{In}_{z} \mathrm{Ga}_{1-z} \mathrm{As}(y=20 \%-24 \%$, $z=10 \%-12 \%$ ) double layer (DL), with a thickness of $300 \mathrm{~nm}$ for each layer. No GaAs cap is deposited on $\mathrm{Ga}(\mathrm{As}, \mathrm{Bi})$ layers. Samples for XPS measurements are protected during the exposure to ambient atmosphere by a capping layer of amorphous As. The whole growth process is monitored in situ by reflection high-energy electron diffraction. Surface morphology is investigated ex situ by a VEECO multimode (Nanoscope IIIa) AFM in the tapping mode by using nonconductive Si tips with a nominal curvature radius of about 5-7 $\mathrm{nm}$.

\section{B. Transmission electron microscopy}

The layers' microstructure is investigated by TEM using a JEOL JEM 3010 electron microscope operated at $300 \mathrm{kV}$. Cross-section TEM specimens are prepared by mechanical polishing, dimpling, and precision ion milling, which is performed with ion-beam energies between 3 and $2.5 \mathrm{keV}$ to minimize Ar-ion milling damage.

\section{X-ray diffraction}

XRD is carried out ex situ utilizing a PANalytical X' Pert PRO MRD diffractometer with Ge (220) hybrid monochromator, employing a $\mathrm{Cu} \mathrm{K} \alpha_{1}$ radiation $(\lambda=1$. $540598 \AA$ ). Reciprocal space maps (RSMs) are acquired around the asymmetric GaAs(224) Bragg peak in coplanar configuration. The $Q_{z}$ and $Q_{y}$ axes are parallel to the GaAs 
TABLE I. Summary of relevant parameters of all the samples grown: composition of the stressor layers (if any), in plane lattice parameter $\left(a_{\mathrm{buffer}}^{\|}\right)$of the buffer layer; in-plane $\left[a_{\mathrm{Ga}(\mathrm{As}, \mathrm{Bi})}^{\|}\right]$and out of plane $\left[a_{\mathrm{Ga}(\mathrm{As}, \mathrm{Bi})}^{\perp}\right]$ lattice parameters, Bi content $\left(x_{\mathrm{Bi}}\right)$ and in plane strain $\left(\varepsilon_{\|}\right)$of $\mathrm{Ga}(\mathrm{As}, \mathrm{Bi})$ film as determined by XRD characterization (see the text). Absolute uncertainties on the determination of the lattice parameters, Bi content, and strain are $0.001 \AA$, 0.2 , and 0.05 , respectively.

\begin{tabular}{|c|c|c|c|c|c|c|c|}
\hline Sample & $(\mathrm{In}, \mathrm{Ga})$ As stressor layer & $a_{\text {buffer }}^{\|}(\AA)$ & $a_{\mathrm{Ga}(\mathrm{As}, \mathrm{Bi})}^{\|}(\AA)$ & $a_{\mathrm{Ga}(\mathrm{As}, \mathrm{Bi})}^{\perp}(\AA)$ & $a_{\mathrm{Ga}(\mathrm{As}, \mathrm{Bi})}(\AA)$ & $x_{\mathrm{Bi}}(\%)$ & $\varepsilon_{||}(\%)$ \\
\hline $\mathrm{C} 1$ & $\mathrm{~N}$ & 5.653 & 5.654 & 5.663 & 5.659 & 0.8 & -0.08 \\
\hline $\mathrm{C} 2$ & $\mathrm{~N}$ & 5.653 & 5.653 & 5.687 & 5.671 & 2.7 & -0.31 \\
\hline $\mathrm{C} 3$ & $\mathrm{~N}$ & 5.653 & 5.654 & 5.705 & 5.681 & 4.1 & -0.49 \\
\hline $\mathrm{C} 4$ & $\mathrm{~N}$ & 5.653 & 5.658 & 5.746 & 5.704 & 7.5 & -0.81 \\
\hline $\mathrm{T} 1$ & $\mathrm{Y}(\mathrm{DL}, y=0.11, z=0.20)$ & 5.706 & 5.704 & 5.617 & 5.658 & 0.7 & 0.81 \\
\hline $\mathrm{T} 2$ & $\mathrm{Y}(\mathrm{DL}, y=0.11, z=0.20)$ & 5.702 & 5.696 & 5.644 & 5.669 & 2.3 & 0.48 \\
\hline $\mathrm{T} 3$ & $\mathrm{Y}(\mathrm{DL}, y=0.10, z=0.21)$ & 5.714 & 5.709 & 5.666 & 5.687 & 4.9 & 0.39 \\
\hline $\mathrm{T} 4$ & $\mathrm{Y}(\mathrm{DL}, y=0.12, z=0.24)$ & 5.720 & 5.718 & 5.685 & 5.700 & 7.0 & 0.31 \\
\hline T5 & $\mathrm{Y}(\mathrm{DL}, y=0.11, z=0.20)$ & 5.706 & 5.705 & 5.699 & 5.702 & 7.2 & 0.06 \\
\hline
\end{tabular}

[001] and [010] directions, respectively. The precise position of the $\mathrm{Ga}(\mathrm{As}, \mathrm{Bi})$ and $(\mathrm{In}, \mathrm{Ga}) \mathrm{As}$ peaks and the data in Table I are determined by fitting the RSM. The uncertainty on the determination of $Q_{y}$ and $Q_{z}$ is less than $0.001 \AA^{-1}$. The data analysis is carried out using $x$-ray utilities [10].

\section{Photoluminescence spectroscopy}

The optical properties of the $\mathrm{Ga}(\mathrm{As}, \mathrm{Bi})$ layers are studied by PL using the 514.5-nm excitation line of a continuous-wave $\mathrm{Ar}^{+}$laser (Coherent Innova Sabre). Samples are mounted in a helium closed cycle cryocooler (Galileo Vacuum Tec), which allows a temperature range from $20 \mathrm{~K}$ to room temperature. PL spectra are collected using a $30-\mathrm{cm}$ monochromator (Acton Research Corporation SpectraPro-300i) with variable slits. Signal detection is accomplished by an amplified and temperature stabilized (In, Ga)As $p$ - $i-n$ photodiode (Hamamatsu G5851-11) with lock-in amplifier technique (Stanford Research Systems SR830 DSP). Typical power density on the sample surface is in the range $10-10^{3} \mathrm{~W} / \mathrm{cm}^{2}$.

\section{E. X-ray photoemission spectroscopy}

XPS is performed on a NanoESCA photoemission microscope (Omicron) with an overall energy resolution of $\Delta E=0.4 \mathrm{eV}$. For excitation we use monochromatized Al $K_{\alpha}$ radiation. To avoid sample surface contamination during the ex situ transport to the NanoESCA UHV chamber, samples are protected by a 100 -nm-thick capping layer of amorphous As. Before carrying out XPS measurements, samples are annealed in UHV at $430{ }^{\circ} \mathrm{C}$ to desorb the protective As cap layer and to obtain the As-rich $2 \times 4$ surface reconstruction [11]. This temperature is high enough to remove possible Bi segregated at the surface and to avoid any effect due to excess Bi on the sample surface [11]. This procedure allows us, by using the $\mathrm{Al} K_{\alpha}$ line, to probe bulklike structures in a region $2-3 \mathrm{~nm}$ from the surface and to rule out a surface origin for the two components identified in the shallow Bi $5 d$ core levels reported in Fig. 6. Spectra are fitted in KolXPD software (http://kolxpd.com) using Voigt peaks on linear background.

\section{F. Raman spectroscopy}

Raman spectra are collected at room temperature. The Stokes scattering of the samples is measured by means of micro-Raman equipment from HORIBA LabRAM HR Evolution. The spectra are excited by a continuous-wave $\mathrm{He}-\mathrm{Ne}$ laser $632.8 \mathrm{~nm}$ equipped with a LN2 cooled CCD detector in backscattering geometry. The emission is focused by a microscope objective with 0.9 numerical aperture and the same objective is used for the collection of the signal. The spectral resolution achieved is $0.7 \mathrm{~cm}^{-1}$ and a notch filter suppressed the stray light. For pure GaAs, the penetration depth of the used Raman laser is about $100 \mathrm{~nm}$, whereas for the $\mathrm{Ga}(\mathrm{As}, \mathrm{Bi})$ alloy it is reduced due to the increase in the absorption coefficient [12]. Thus, the thicknesses of the studied samples allow for the study of the $\mathrm{Ga}(\mathrm{As}, \mathrm{Bi})$ topmost layer.

\section{G. Density-functional theory}

Calculations based on density functional theory (DFT) are carried out using the Quantum Espresso suite [13,14] to study the structural and electronic properties of the $\mathrm{GaAs}_{1-x} \mathrm{Bi}_{x}$ alloys as a function of the buffer-layer lattice parameter. Norm-conserving core-corrected not relativistic pseudopotentials are used for the $\mathrm{Ga}$ and As atoms, whereas a full relativistic pseudopotential is used for the $\mathrm{Bi}$ atom. The local-density approximation (LDA) as parametrized by Perdew and Zunger [15] is used for the exchange-correlation potential. The pseudopotentials are tested on a series of GaAs-based materials and on the $\mathrm{Bi}$ metal, obtaining structural parameters in agreement with the experiment. The electron wave functions and the charge density are expanded in plane waves, whose energy is less than 45 Ry and 360 Ry for the wave functions and the charge density, respectively. To integrate the charge density, we use grids of $k$ points corresponding 
to 8 and $16 k$ points in the irreducible part of the Brillouin zone. The $\mathrm{Ga}(\mathrm{As}, \mathrm{Bi})$ alloys are modeled through unit cells containing 128 atoms whose in-plane ( $x$ and $y$ ) lattice constant is fixed at three different values: the calculated equilibrium lattice constant of GaAs, and two other lattice constants, one $0.83 \%$ and the other $1.4 \%$ larger than the GaAs lattice constant. Such in-plane lattice constants correspond to the experimental in-plane lattice parameter of the partially relaxed (In, Ga)As DL and to a completely relaxed (In, Ga)As DL. The vertical cell dimension along $z$ and the atomic positions are simultaneously optimized until the forces are less than $0.01 \mathrm{eV} / \AA$. For each inplane lattice parameter we consider cells containing one Bi (concentration $1.5625 \%$ ), two $\mathrm{Bi}(x=3.125 \%)$, and five $\mathrm{Bi}(x=7.8125 \%)$. The $\mathrm{Bi}$ atoms are distributed among the sites, substitutional to As, in different ways. We do not consider here configurations with $\mathrm{Bi}$ atoms in firstneighbor positions, where $\mathrm{Bi}$ is substitutional also to $\mathrm{Ga}$ atoms.

\section{RESULTS AND DISCUSSION}

\section{A. Tetragonal deformation of strained $\mathrm{Ga}(\mathrm{As}, \mathrm{Bi})$ films}

The main goal of the present paper is the strain engineering of the pseudomorphic growth of $\mathrm{Ga}(\mathrm{As}, \mathrm{Bi})$ on GaAs(001) by MBE and the use of appropriate intermediate buffer layers acting as stressors; in this regards, two sets of samples are prepared with the $\mathrm{Ga}(\mathrm{As}, \mathrm{Bi})$ layers under compressive and tensile conditions, respectively (Fig. 1). The first set of samples consists of four different $\mathrm{GaAs}_{1-x} \mathrm{Bi}_{x}$ films ( $x$ ranging from 0.83 to $7.5 \%$ )

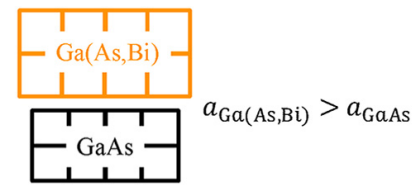

C1-C4 set:

\begin{tabular}{c}
$\mathrm{GaAs}_{1-x} \mathrm{Bi}_{x}$ \\
\hline $\mathrm{GaAs}$ \\
\hline
\end{tabular}

Compressive strain

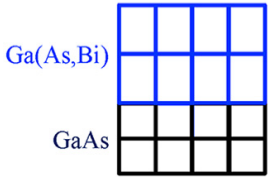

T1-T5 set:

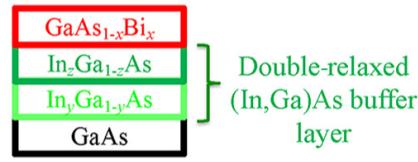

Tensile strain

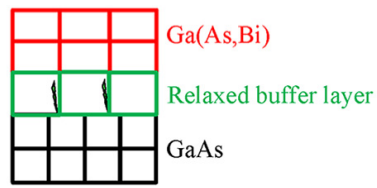

FIG. 1. Schematic of the growth approach employed in this work. By using appropriate partially relaxed double buffer layers of (In, Ga)As, films of $\mathrm{Ga}(\mathrm{As}, \mathrm{Bi})$ under tensile strain are grown and studied in comparison with standard $\mathrm{Ga}(\mathrm{As}, \mathrm{Bi}$ ) films directly grown on $\mathrm{GaAs}$ under compressive strain. grown under compressive strain on the GaAs buffer layer (denoted as $\mathrm{C} 1, \mathrm{C} 2, \mathrm{C} 3$, and $\mathrm{C} 4$, see Table I). The second set is composed of five samples (T1, T2, $\mathrm{T} 3$, T4, and $\mathrm{T} 5$, see Table I), where the $\mathrm{GaAs}_{1-x} \mathrm{Bi}_{x}$ $(x=0.74 \%-7.2 \%)$ layers are grown under tensile strain on top of a partially relaxed $\mathrm{In}_{y} \mathrm{Ga}_{1-y} \mathrm{As} / \mathrm{In}_{z} \mathrm{Ga}_{1-z} \mathrm{As}$ $(y=20 \%-24 \%, z=10 \%-12 \%)$ DL, with a thickness of $300 \mathrm{~nm}$ for each layer. All the samples were characterized by XRD in order to ascertain their crystal quality, and to determine the Bi content. As an example, Figs. 2(a) and 2(b) show the RSMs around the GaAs(224) Bragg
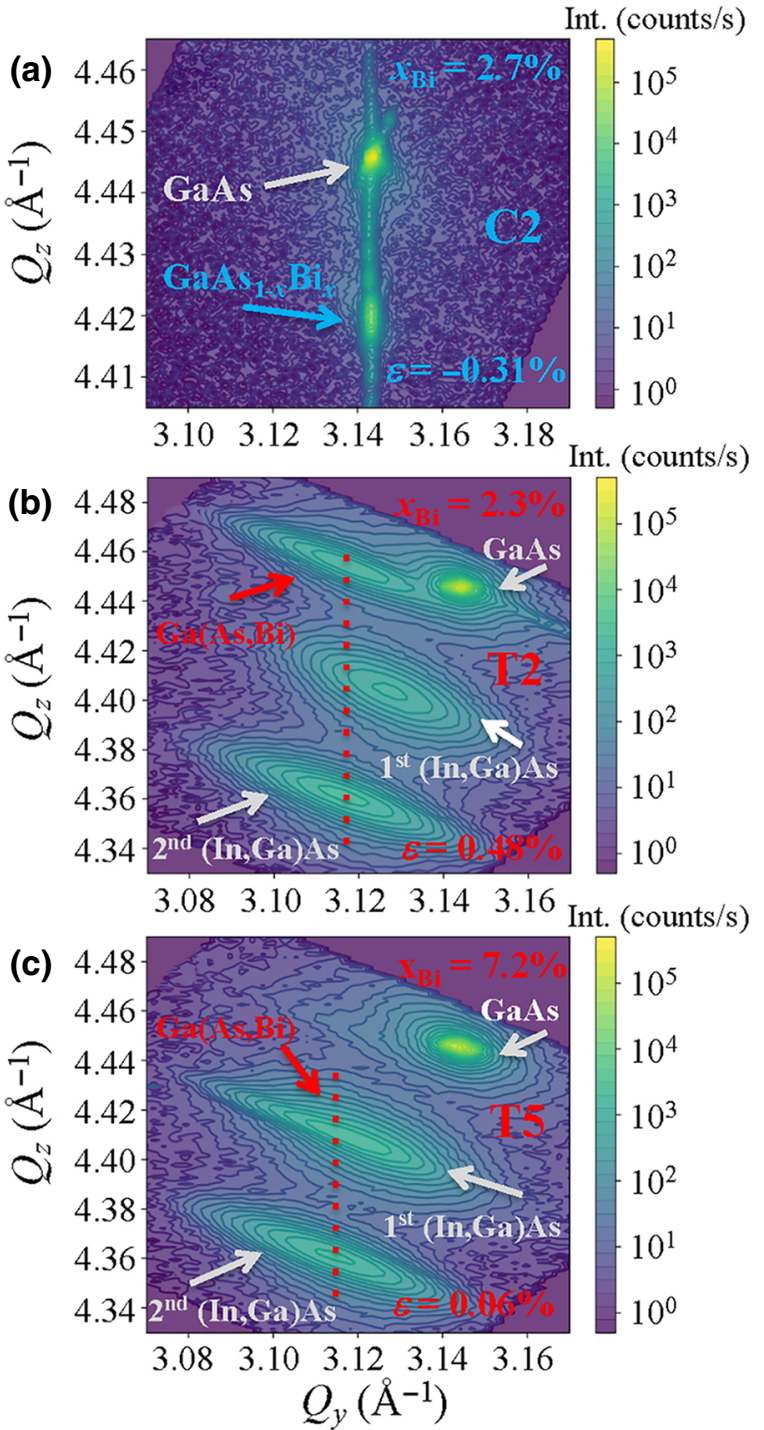

FIG. 2. Reciprocal space maps of three representative samples: (a) $\mathrm{Ga}(\mathrm{As}, \mathrm{Bi})$ layer under compressive strain (C2) on a GaAs substrate, (b) Ga(As, Bi) layer under tensile strain (T2) grown on a partially relaxed $\mathrm{In}_{0.2} \mathrm{Ga}_{0.8} \mathrm{As} / \mathrm{In}_{0.1} \mathrm{Ga}_{0.9}$ As double layer, and (c) $\mathrm{Ga}$ (As, Bi) layer grown under quasimatched conditions (T5) on a partially relaxed $\operatorname{In}_{0.2} \mathrm{Ga}_{0.8} \mathrm{As} / \mathrm{In}_{0.1} \mathrm{Ga}_{0.9}$ As double layer. Red dotted lines highlight the final $Q_{y}$ position of the second (In, Ga)As layer. 
reflection for $\mathrm{Ga}(\mathrm{As}, \mathrm{Bi})$ layers with an intermediate $\mathrm{Bi}$ content, $\mathrm{C} 2$ and $\mathrm{T} 2$, under compressive and tensile strain conditions, respectively. Figure 2(c) shows the same map for a sample (T5) grown with a high Bi content but in unstrained, so-called "quasimatched" conditions. The map in Fig. 2(a) shows two intense peaks for the GaAs substrate and $\mathrm{Ga}(\mathrm{As}, \mathrm{Bi})$ film, while the fringes, clearly visible along $Q_{z}$, denote the high epitaxial quality of the interface, typical of coherent epilayers. $\mathrm{Ga}(\mathrm{As}, \mathrm{Bi}$ ) and $\mathrm{GaAs}$ peaks exhibit the same in-plane component $Q_{y}$ of the scattering vector. This means that the $\mathrm{Ga}(\mathrm{As}, \mathrm{Bi})$ film has the same in-plane lattice parameter as the GaAs substrate, i.e., it is pseudomorphic to GaAs and therefore under compressive strain. It undergoes a tetragonal distortion with an out-of-plane lattice parameter larger than the in-plane one, as calculated (see below) by the value of the $Q_{z}$ component (smaller than that of GaAs). This behavior is common to all the samples directly grown on GaAs, even for the highest Bi contents (see Table I). A virtually negligible relaxation can be detected for the $\mathrm{C} 4$ sample (not shown): the inplane lattice parameter is in fact a few thousandths of $\AA$ greater than that of GaAs, indicating a just incipient plastic relaxation. A different scenario appears if we look at Fig. 2(b), where the RSM of the sample grown with the same conditions as sample $\mathrm{C} 2$ but on top of the (In, Ga)As $\mathrm{DL}$ is shown. In this case, four peaks are detected, one for the GaAs substrate, two for the (In, Ga)As layers, and one for the $\mathrm{Ga}(\mathrm{As}, \mathrm{Bi})$ film. It is evident that both (In, Ga)As layers undergo a partial plastic relaxation with the corresponding peaks placed at lower $Q_{y}$ and $Q_{z}$ values than that of the GaAs peak. Most importantly, the Ga(As, Bi) peak is now vertically aligned to the second ( $\mathrm{In}, \mathrm{Ga}$ ) As layer, therefore matching an in-plane lattice parameter larger than that of GaAs (see Table I and the lattice parameters' determination below). For all the $\mathrm{Ga}$ (As, Bi) layers grown onto an (In, Ga)As DL a minimal (negligible) relaxation is detected, with the in-plane $\mathrm{Ga}(\mathrm{As}, \mathrm{Bi})$ lattice parameter resulting in a few thousandths of $\AA$ smaller than that of the second (In, Ga)As layer (see below). The only exception is represented by sample T5 [whose RSM is shown in Fig. 2(c)], which is quasilattice matched to the relaxed (In, Ga)As DL. It represents an example of $\mathrm{Ga}(\mathrm{As}, \mathrm{Bi}$ ) with a high Bi content (7.2\%) but essentially unstrained (see Table I and the lattice parameters' determination below). In general, the relaxation observed for the samples under tensile strain is very small, in particular when increasing the $\mathrm{Bi}$ content (as the tensile strain reduces). Therefore, the growth can be considered almost pseudomorphic to the second (In, Ga)As layer. This makes the in-plane lattice parameter of $\mathrm{Ga}(\mathrm{As}, \mathrm{Bi})$ large enough to invert the sign of the tetragonal distortion of $\mathrm{Ga}(\mathrm{As}, \mathrm{Bi})$ with respect to the first set of samples: on the (In, Ga)As $\mathrm{DL}, \mathrm{Ga}(\mathrm{As}, \mathrm{Bi})$ alloy is under biaxial tensile stress in the (001) plane, which causes a lattice-parameter shortening along the growth direction.
The analysis of the RSMs allows us to determine the inplane and out-of-plane lattice parameters of all the samples (Table I). The tetragonal strain relation:

$$
\varepsilon_{\perp}=\frac{-2 v}{1-v} \varepsilon_{\|},
$$

with $v$ being Poisson's coefficient, relates the in-plane $\left(\varepsilon_{\|}\right)$ and out-of-plane $\left(\varepsilon_{\perp}\right)$ strain components of a cubic lattice under an in-plane biaxial stress. $\varepsilon_{\|}$and $\varepsilon_{\perp}$ are defined as

$$
\varepsilon_{\|}=\frac{a_{\mathrm{Ga}(\mathrm{As}, \mathrm{Bi})}^{\|}-a_{\mathrm{Ga}(\mathrm{As}, \mathrm{Bi})}}{a_{\mathrm{Ga}(\mathrm{As}, \mathrm{Bi})}}, \quad \varepsilon_{\perp}=\frac{a_{\mathrm{Ga}(\mathrm{As}, \mathrm{Bi})}^{\perp}-a_{\mathrm{Ga}(\mathrm{As}, \mathrm{Bi})}}{a_{\mathrm{Ga}(\mathrm{As}, \mathrm{Bi})}},
$$

where $a_{\mathrm{Ga}(\mathrm{As}, \mathrm{Bi})}^{\|}$and $a_{\mathrm{Ga}(\mathrm{As}, \mathrm{Bi})}^{\perp}$ are the measured in-plane and out-of-plane lattice parameters, respectively, of the tetragonally distorted $\mathrm{Ga}(\mathrm{As}, \mathrm{Bi})$ cell and $a_{\mathrm{Ga}(\mathrm{As}, \mathrm{Bi})}$ the equilibrium lattice parameter of the related cubic cell. By means of Eqs. (1) and (2) we first determine $a_{\mathrm{Ga}(\mathrm{As}, \mathrm{Bi})}$ approximating the $\mathrm{Ga}(\mathrm{As}, \mathrm{Bi})$ Poisson's ratio with the one of GaAs. Then, applying Vegard's law: $a_{\mathrm{Ga}(\mathrm{As}, \mathrm{Bi})}=$ $x a_{\mathrm{GaBi}}+(1-x) a_{\mathrm{GaAs}}$, we obtain the Bi content $x$ for each sample (Table I). We use the literature value $a_{\mathrm{GaAs}}=$ $5.6532 \AA$ for GaAs [16]. The binary compound GaBi has not yet been grown, therefore we use the lattice parameter $a_{\mathrm{GaBi}}=6.33 \AA$, compatible with both theoretical calculations and experimental estimations in the literature [7,17]. The resulting $\varepsilon_{\|}$for each sample are reported in Table I, where the absolute uncertainties on the determination of the lattice parameters, strain, and Bi content are, $0.001 \AA$, 0.05 , and 0.2 , respectively. As expected, for the $\mathrm{Ga}(\mathrm{As}, \mathrm{Bi})$ films grown on GaAs the compressive strain on the alloy increases with the $\mathrm{Bi}$ content. On the other hand, for $\mathrm{Ga}$ (As, Bi) films grown under tensile strain (second set of samples), the strain instead decreases with the Bi content.

In Fig. 3, we compare the measured tetragonal distortion $(t)$ in the $\mathrm{Ga}(\mathrm{As}, \mathrm{Bi})$ films with the results of DFT calculations. The tetragonal distortion, being defined as

$$
t=\frac{a_{\mathrm{Ga}(\mathrm{As}, \mathrm{Bi})}^{\perp}-a_{\mathrm{Ga}(\mathrm{As}, \mathrm{Bi})}^{\|},}{a_{\mathrm{Ga}(\mathrm{As}, \mathrm{Bi})}^{\|}}
$$

is plotted as a function of the $\mathrm{Bi}$ content $x$. The calculated values (circles) are shown for three different buffer-layer lattice constants: $5.653 \AA$ (GaAs, blue symbols), $5.701 \AA$ [partially relaxed (In, Ga)As DL, red symbols], and $5.732 \AA$ [completely relaxed (In, Ga)As DL, green symbols], where the DFT-LDA calculated parameters [slightly underestimated $(0.85 \%)$ with respect to the experiment] are reported in the figure rigidly shifted to the experimental ones. The agreement between the measured parameters (blue and red triangles) and the calculated ones (blue and red dots) is remarkable [we point out that sample 


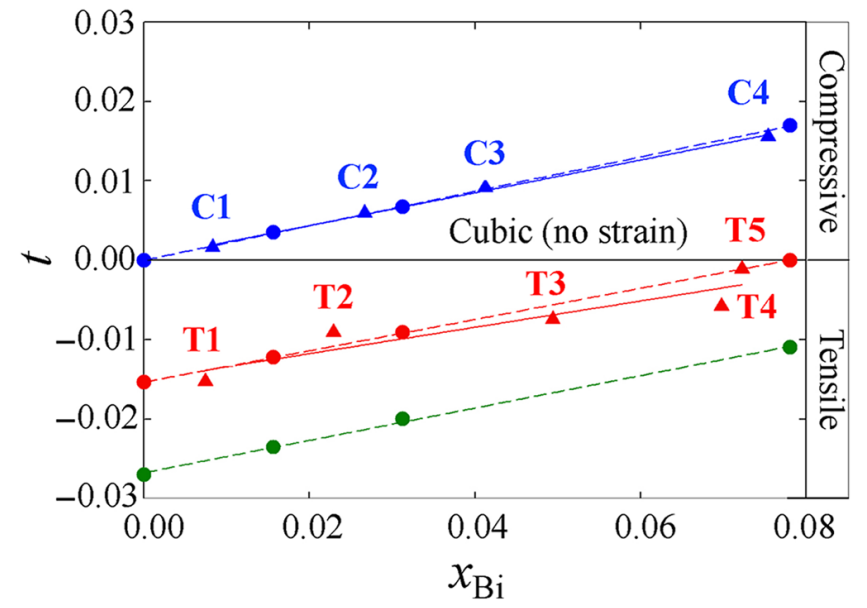

FIG. 3. Comparison between the experimental tetragonal distortion $t$ in $\mathrm{Ga}(\mathrm{As}, \mathrm{Bi})$ films under compressive (blue triangles) and under tensile (red triangles) strain with the results of DFT calculations (blue dots and red dots for compressive and tensile strain, respectively). Continuous and dashed lines are linear fits to the experimental and theoretical data, respectively. Green symbols and line represent theoretical data and fit for the case of a completely relaxed $\operatorname{In}_{y} \mathrm{Ga}_{1-y} \mathrm{As} / \mathrm{In}_{z} \mathrm{Ga}_{1-z} \mathrm{As}$ double buffer layer.

T4 is the only one grown on a different (In, Ga)As DL]. The value of $t$ does not depend significantly on the configuration of the $\mathrm{Bi}$ atoms in the matrix, isolated atoms, or atoms located in neighboring sites-second neighbors on the zinc-blende lattice produce the same tetragonal deformation of the lattice. This result also validates the determination of the alloy concentration by using the in-plane and out-of-plane strains, as determined by XRD.

Starting from Eqs. (1) and (2) and applying Vegard's law, we can determine the expected behavior of $t$ as a function of the Bi content $x$, which reads as

$t=(1+k)\left(\frac{a_{\mathrm{GaAs}}}{a_{\mathrm{Ga}(\mathrm{As}, \mathrm{Bi})}^{\|}}-1\right)+\left[\frac{1+k}{a_{\mathrm{Ga}(\mathrm{As}, \mathrm{Bi})}^{\|}}\left(a_{\mathrm{GaBi}}-a_{\mathrm{GaAs}}\right)\right] x$,

where $k=[2 v /(1-v)]$. By approximating the $\mathrm{Ga}(\mathrm{As}, \mathrm{Bi})$ Poisson's ratio as a constant (the one of GaAs), the tetragonal distortion $t$ is linear with $x$, the slope being dependent on the in-plane lattice parameter $a_{\mathrm{Ga}(\mathrm{As}, \mathrm{Bi})}^{\|}$. For the values of the buffer-layer lattice parameter $a_{\mathrm{Ga}(\mathrm{As}, \mathrm{Bi})}^{\|}$considered in this paper, such dependence is very small and almost negligible, with the expected slopes ranging from 0.227 to 0.224 . As a matter of fact, the fit of the calculated values of $t$, using a linear dependence $t(x)=t(0)+q x$, is remarkably good and gives the values of the slopes: $q=0.217$ for the GaAs buffer layer (blue symbols), $q=0.197$ for the partially relaxed (In, Ga)As DL (red symbols), and $q=$ 0.204 for the largest in-plane lattice constant (green symbols). Similar values of $q$ are found fitting the experimental data. The slightly larger deviation between experimental and calculated slopes at higher Bi concentration in the case of the tensile samples is due to the inclusion in the fit of $\mathrm{T} 4$, which is grown on a DL with more In and exhibits a larger in-plane lattice parameter (see Table I). Although there is no experimental counterpart for the completely relaxed DL, this result shows that it is possible to predict the tetragonal deformation of the $\mathrm{Ga}(\mathrm{As}, \mathrm{Bi})$ as a function of the Bi concentration $x$.

By comparing the data in Table I with Fig. 3, we note that films deposited with the same growth parameters [in particular the same Bi flux, i.e., the couples C1-T1, C2-T2, C4-T4-(T5)], but under different strain conditions, exhibit a reduced $\mathrm{Bi}$ incorporation (about 10\%) when the strain goes from compressive to tensile. For example, sample T5 is quasi-lattice-matched to the DL and its $\mathrm{Bi}$ content is lower than that of sample $\mathrm{C} 4$, which is under a compressive strain, but larger than that of sample T4, subjected to a tensile strain. Thus, we find a higher Bi incorporation in the compressively strained samples than in the tensely strained ones, contrary to our expectation (see below).

Our DFT calculations define the stability of substitutional $\mathrm{Bi}$ atoms (Bi substituting $\mathrm{As}$ ) in the GaAs matrix in terms of the quantity:

$$
E_{\mathrm{misc}}\left(a^{\|}\right)=E\left(a^{\|}\right)-N_{\mathrm{As}} \mu_{\mathrm{GaAs}}\left(a^{\|}\right)-N_{\mathrm{Bi}} \mu_{\mathrm{GaBi}}\left(a^{\|}\right),
$$

which is a measure of the miscibility of Bi inside GaAs. In this expression $E\left(a^{\|}\right)$is the total energy of the $\mathrm{Ga}(\mathrm{As}, \mathrm{Bi})$ alloy with a number $N_{\mathrm{As}}$ of arsenic atoms and a number $N_{\mathrm{Bi}}$ of bismuth atoms in the unit cell. $a^{\|}$is the in-plane lattice parameter. $\mu_{\mathrm{GaAs}}$ and $\mu_{\mathrm{GaBi}}$ are the total energies per Ga-As and Ga-Bi pair of the binary GaAs and $\mathrm{GaBi}$ systems. All the total energies are calculated for the same value of $a^{\|}$. If $E_{\text {misc }}$ is positive the system will tend to phase segregate, the contrary is true if $E_{\text {misc }}$ is negative. The calculation investigates the thermodynamic tendency to mix or segregate, and is valid only if the growth conditions are close to the thermodynamic limit (high temperatures and slow growth rates). Our calculations produce in all cases $E_{\text {misc }}>0$, with the largest values of $E_{\text {misc }}$ occurring when $a^{\|}=a_{\text {GaAs. }}$. These values of $E_{\text {misc }}$ indicate that the substitution of $\mathrm{As}$ with $\mathrm{Bi}$ is not energetically favorable and they agree with the observation of Bi surface segregation during growth [6,7]. $E_{\text {misc }}$ decreases of $27-28 \mathrm{meV}$ per cell and per Bi atom when the substrate lattice parameter is increased by $1.4 \%$. Thus, the calculations predict that the miscibility could be slightly improved increasing the substrate lattice parameter. This result agrees with a recent theoretical work [18] that predicts an increase of 2 orders of magnitude of Bi solubility when Ga(As, Bi) is epitaxially constrained to an in-plane lattice parameter $5 \%$ larger than that of bulk GaAs. Thus, one would expect a slightly increased $\mathrm{Bi}$ incorporation in the case of the samples grown on the DL buffer, differently from 
our experimental result. This discrepancy can be explained considering that the theoretical calculations apply to the thermodynamic limit, while in our experiments, due to the low growth temperature, the alloys are grown in conditions very far away from equilibrium. In this regime, kinetic effects are dominant and $\mathrm{Bi}$ incorporation is affected by the ratios of the rates of deposition, diffusion, and desorption of both cations and anions. Interestingly, we observe a reduced $\mathrm{Bi}$ incorporation when the growth is carried out under tensile-stress conditions.

\section{B. Characterization of crystal quality}

The surface morphology of our samples clearly testifies the different strain conditions between the two sets of samples, as shown in Fig. 4, where the ex situ atomic force microscopy (AFM) topographies of the two couples of samples $\mathrm{C} 2-\mathrm{T} 2$ and $\mathrm{C} 4-\mathrm{T} 4$ (similar Bi content but different strain conditions) are reported. Sample C2 [Fig. 4(a)] exhibits a flat surface [surface roughness $\left.\sigma_{\text {rms }}(\mathrm{C} 2)=0.30 \mathrm{~nm}\right]$ completely free of Bi-related surface features and/or droplets, a clear evidence of good epitaxy conditions for our samples. This is the case for all the samples grown under compressive strain, except for sample $\mathrm{C} 4$
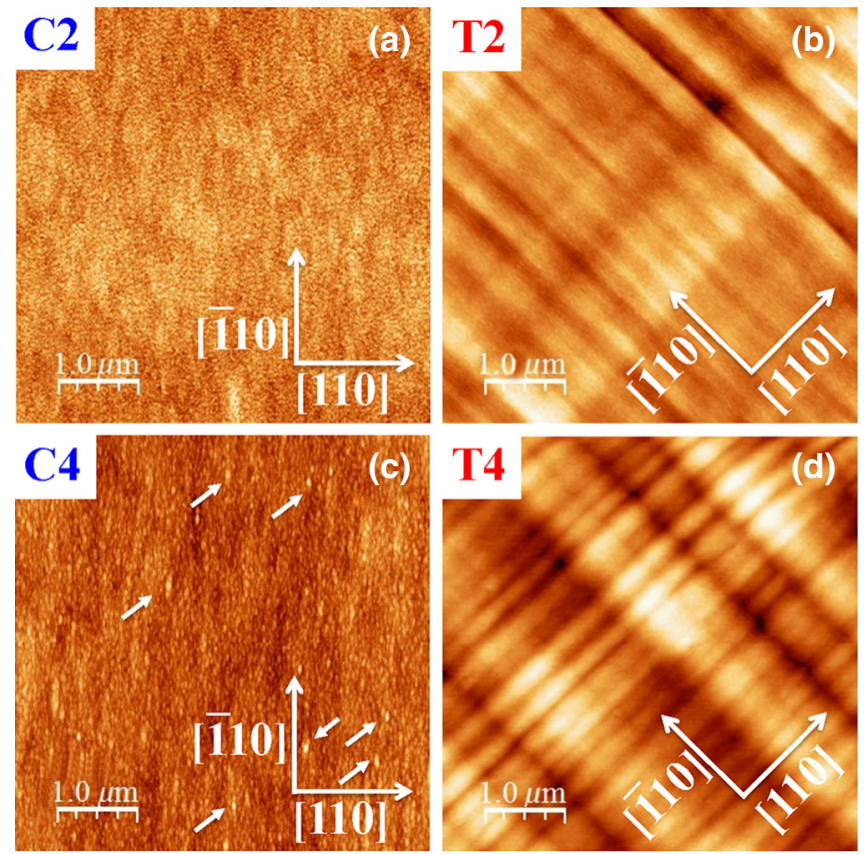

FIG. 4. AFM topographies of two couples of $\mathrm{Ga}(\mathrm{As}, \mathrm{Bi})$ samples (C2-T2 and C4-T4) with similar $\mathrm{Bi}$ concentrations but different strain conditions: compressive (a), (c) and tensile (b), (d). The surface is always free from Bi cluster, apart for high $\mathrm{Bi}$ content and high compressive strain (C4), where the presence of small clusters is indicated by white arrows. The texture present in tensile-strained samples is related to the occurrence of crosshatch patterns due to the misfit dislocations in the (In, Ga)As double layer.
[Fig. 4(c)], which incorporates the highest Bi concentration $\left(x_{\mathrm{Bi}}=7.5 \%\right)$. In this case, a low density of small Bi-related features (about $5 \mathrm{~nm}$ high, on average) can be observed on the surface [surface roughness $\sigma_{\mathrm{rms}}(\mathrm{C} 4)=0.86 \mathrm{~nm}$ ].

The morphology of the films grown on the (In, Ga)As DL [Figs. 4(b) and 4(d)] is completely different. The surface of samples T2 and T4 shows a cross-hatched (CH) morphology made of undulations $2-4 \mathrm{~nm}$ high, which significantly increase the average surface roughness $\left[\sigma_{\text {rms }}(\mathrm{T} 2)=1.2 \mathrm{~nm}, \sigma_{\text {rms }}(\mathrm{T} 4)=1 \mathrm{~nm}\right]$ compared to that of the samples directly grown on GaAs. The formation of such a $\mathrm{CH}$ pattern is well known in the literature [19-21] as a footprint of long segments of interfacial misfit dislocations (MDs) originating upon plastic relaxation of the (In, Ga)As film in the case of low mismatch. Therefore, all the samples grown under tensile conditions (T1-T5) exhibit the $\mathrm{CH}$ pattern. However, this is not an indication of low-quality $\mathrm{Ga}(\mathrm{As}, \mathrm{Bi})$ material, as shown by TEM. The images reported in Fig. 5 are chemically sensitive dark-field TEM (DF) images of the samples $\mathrm{C} 2$, T2, and T5 obtained with the diffraction vector $\mathbf{g}=002$. This imaging mode is highly sensitive to variations in the chemistry of the alloy in semiconductors with zinc-blende (ZB) structure [22]. Furthermore, $\mathbf{g}_{002}$ DFTEM is a powerful technique to detect composition fluctuations in $\mathrm{Ga}(\mathrm{As}, \mathrm{Bi})$ [23]. The representative images shown in Fig. 5 evidence the high compositional homogeneity of the samples, regardless of the strain state of the layer, i.e., compressive (C2), tensile (T2), and quasi-lattice-matched (T5), where no composition fluctuations or $\mathrm{Bi}$ cluster formation are detected at this length scale (approximately equals $100 \mathrm{~nm}$ ), even in the case of the highest $\mathrm{Bi}$ content $\left(x_{\mathrm{Bi}}=7.2 \%\right.$, sample T5). The comparison of the TEM images for the tensile and compressive samples demonstrates the possibility to control the $\mathrm{Ga}(\mathrm{As}, \mathrm{Bi})$ strain condition without affecting the crystal quality. Additionally, analysis of the $\mathbf{g}_{002}$ diffracted intensity enables quantification of the chemical composition [22]. We find a fair agreement between the rough estimation of the $\mathrm{Bi}$ content based on TEM $\left[x_{\mathrm{Bi}}(\mathrm{C} 2) \sim 2.4 \%, x_{\mathrm{Bi}}(\mathrm{T} 2) \sim 2.1 \%, x_{\mathrm{Bi}}(\mathrm{T} 5) \sim 6.9 \%\right]$ and that determined using XRD (Table I), which reinforces the suitability of $\mathbf{g}_{002}$ DFTEM for the analysis of $\mathrm{Ga}(\mathrm{As}, \mathrm{Bi}$ ) layers $[23,24]$ (more details on TEM analysis can be found in Appendix A).

To investigate the presence of nonequivalent incorporation sites for $\mathrm{Bi}$ atoms, we study, by means of highresolution x-ray photoemission spectroscopy (XPS), two different samples grown in the same conditions, namely $\mathrm{C} 4$ $\left(\mathrm{C} 4 b-x_{\mathrm{Bi}}=7.5 \%\right.$, compressive) and $\mathrm{T} 5\left(\mathrm{~T} 5 b-x_{\mathrm{Bi}}=7.2 \%\right.$, quasimatched). The analysis of the Bi $5 d$ and $4 f$ core levels presented in Fig. 6 clearly shows, within our resolution, for both samples, two different components $[\operatorname{Bi}(1)$ and $\mathrm{Bi}(2)]$ that can be ascribed to the presence of at least two nonequivalent $\mathrm{Bi}$ sites in the GaAs lattice. The presence of 

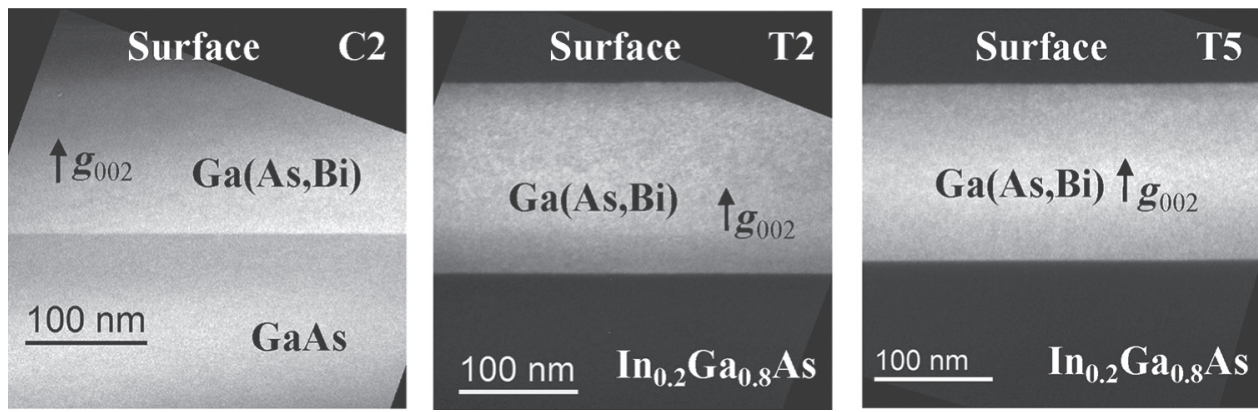

FIG. 5. Chemically sensitive go02 cross-section TEM-DF images of representative $\mathrm{Ga}(\mathrm{As}, \mathrm{Bi})$ layers grown under compressive $(\mathrm{C} 2)$, tensile strain (T2), and quasimatched (T5) conditions. The images show the high compositional homogeneity and the absence of dislocations on a range of several hundreds of nm. a third component due to segregated metallic $\mathrm{Bi}$, expected at a slightly higher binding energy than the $\mathrm{Bi}(1)$ and $\mathrm{Bi}(2)$ components $[25,26]$, is not detected for both the samples. Therefore, we can conclude that the $\mathrm{Bi}(1)$ and $\mathrm{Bi}(2)$ corelevel components originate from different $\mathrm{Bi}-\mathrm{Ga}$ bonding configurations due to $\mathrm{Bi}$ complexes [26] formed by one, two, or three fcc Bi nearest neighbors to the same Ga atom, or by Bi-Ga-Bi-Ga-Bi chains along given crystal directions. It is reasonable to assume that the lower binding energy component $\mathrm{Bi}(1)$, more intense, might be related to isolated substitutional $\mathrm{Bi}$ atoms, while $\mathrm{Bi}(2)$, less intense and slightly more bonded, to some Bi-Ga-Bi complexes. The $\mathrm{C} 4 b$ and T5b samples show a different ratio in the relative intensity of these two components, which represents a different relative concentration of the two nonequivalent $\mathrm{Bi}$ atoms in the two samples. Also, we need to remember that T5 is almost unstrained, suggesting that the in-plane compressive strain favors the formation of complexes, even according to DFT calculations that predict a larger miscibility gap under compressive strain. This result gives evidence of the presence of different $\mathrm{Bi}$ configurations in a different proportion in the $\mathrm{Ga}(\mathrm{As}, \mathrm{Bi})$ films grown under a different epitaxial strain. Further insights on the structural properties of the $\mathrm{Ga}(\mathrm{As}, \mathrm{Bi})$ films are obtained by means of Raman spectroscopy (see Appendix B), which indicates a higher crystal order for the $\mathrm{Ga}(\mathrm{As}, \mathrm{Bi})$ tensile samples with respect to the compressive ones.

Summarizing, both AFM and TEM show no evidence of Bi clusters at the surface (except sample C4) or phase separation (Figs. 4 and 5) in our $\mathrm{Ga}(\mathrm{As}, \mathrm{Bi})$ samples. XPS and Raman spectroscopies hint at a more uniform crystalline structure in the case of the samples grown under tensile biaxial stress in terms of both composition (minor number of Bi complexes) and of long-range lattice order.

\section{Strain-dependent photoluminescence properties}

Figure 7 shows the normalized low-temperature PL spectra collected at the same excitation power for the three couples of $\mathrm{Ga}(\mathrm{As}, \mathrm{Bi})$ samples, each couple with a similar Bi content but under different strain conditions: compressive versus tensile. The low-temperature PL peak
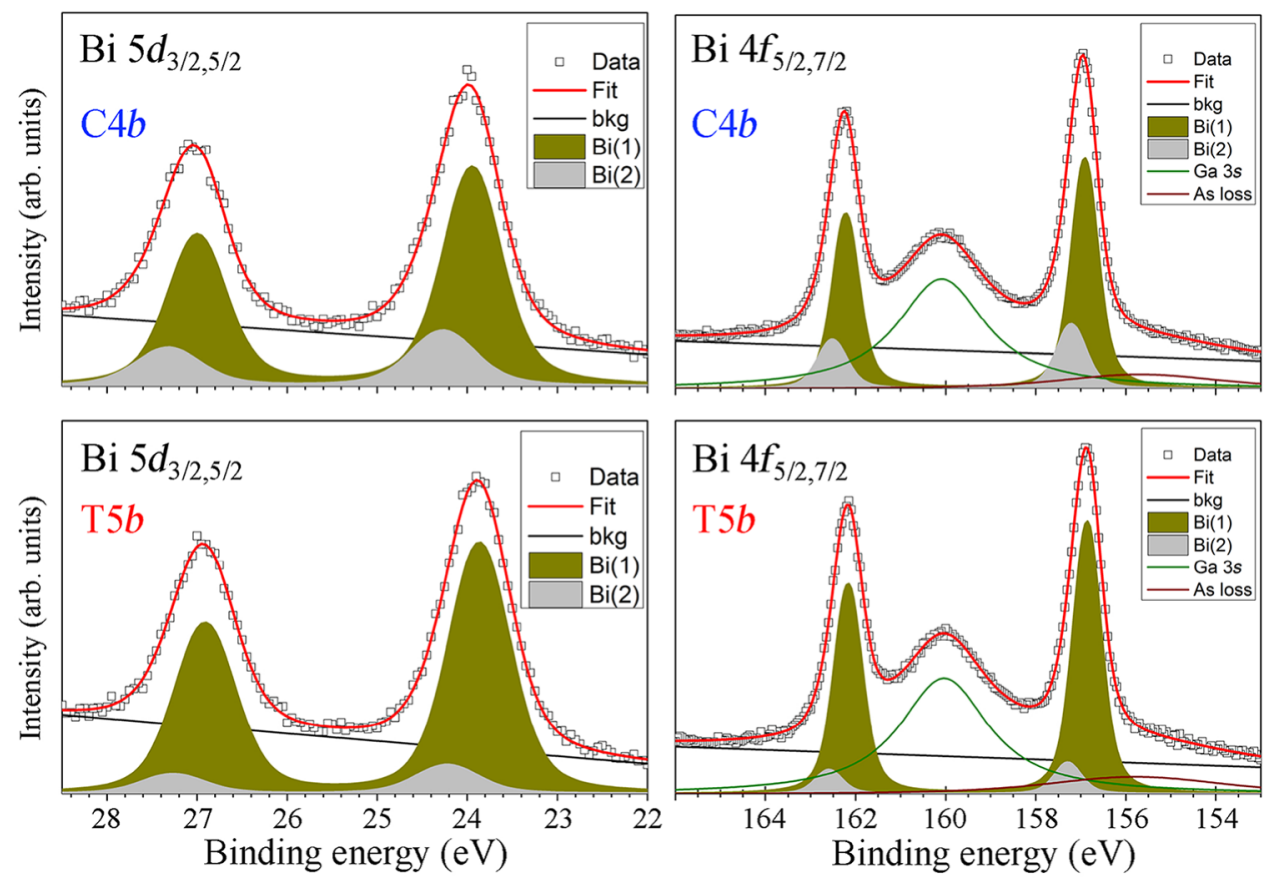

FIG. 6. Bi $5 d$ and $4 f$ XPS spectra of corresponding $\mathrm{Ga}(\mathrm{As}, \mathrm{Bi})$ layers grown under compressive $(\mathrm{C} 4 b)$ and under quasi-matched conditions $(\mathrm{T} 5 b)$. In both cases, we find, together with the main component due to Ga-Bi bound [Bi(1)], a higher binding energy component $[\mathrm{Bi}(2)]$ with a low intensity that is likely due to $\mathrm{Bi}$ Ga-Bi complexes. 

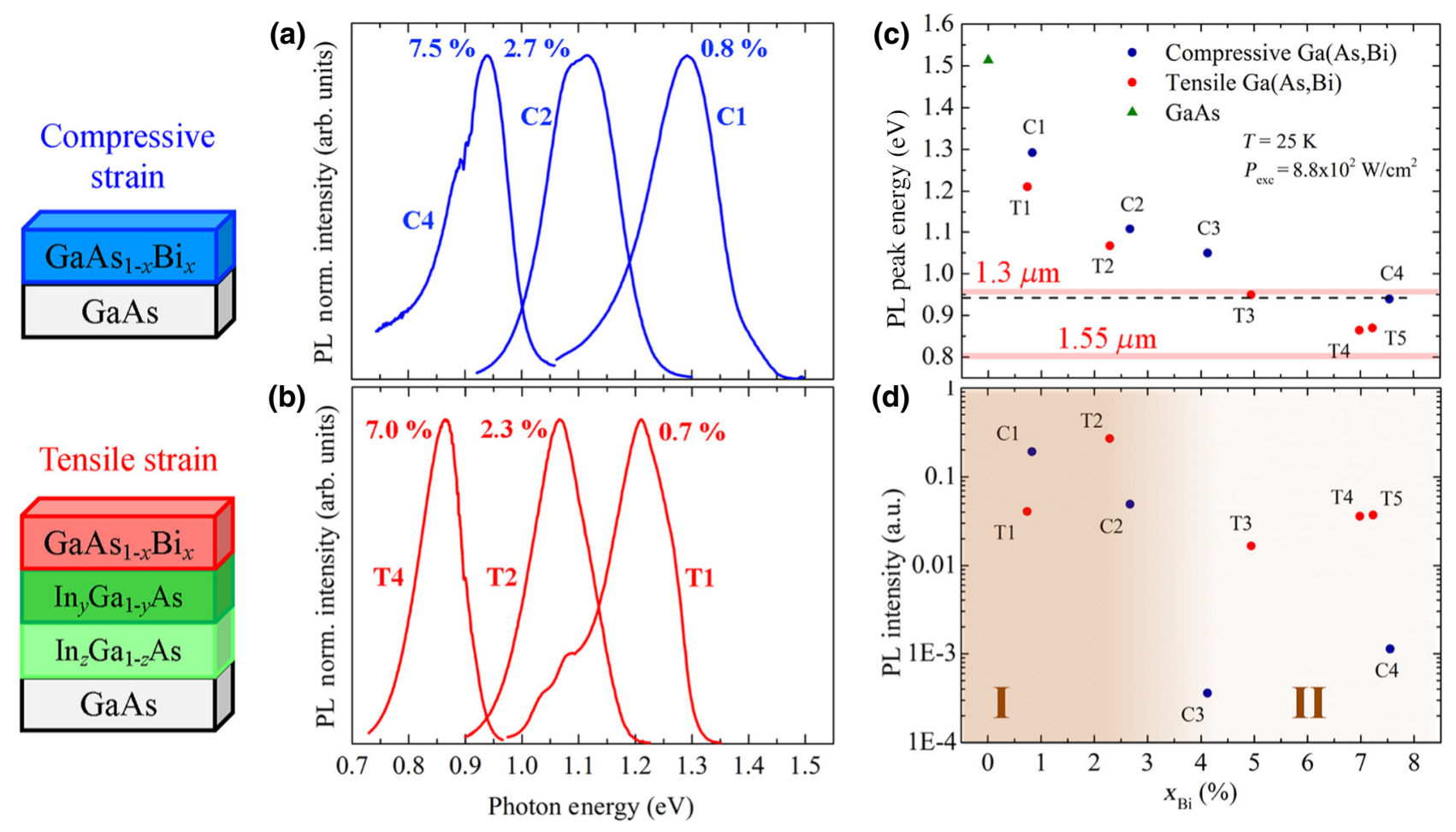

FIG. 7. Normalized PL spectra taken at the same conditions and excitation power density $W=8.8 \times 10^{2} \mathrm{~W} / \mathrm{cm}^{2}$ for compressive (a) and tensile (b) $\mathrm{Ga}(\mathrm{As}, \mathrm{Bi}$ ) layers. (c) PL peak energy plotted as a function of Bi concentration. The dashed line highlights that the emission at $1.3 \mu \mathrm{m}$ is achieved in tensile samples with $35 \%$ less Bi than the compressive ones; the light red stripes show the most important telecommunication bands $(1.3$ and $1.55 \mu \mathrm{m}$ ). (d) Absolute PL intensity plotted as a function of Bi concentration; the two light brown areas help to highlight the low-intermediate (I) and high-intermediate (II) Bi content regions to better show the higher quality of tensile samples.

for the standard compressive samples $\mathrm{C} 1, \mathrm{C} 2$, and $\mathrm{C} 4$, appears redshifted with respect to the expected free exciton [27-31]. Such a redshift is well known in this material and is explained by the presence of Bi-related localized states close to the valence-band maximum (VBM), which dominates the exciton recombination processes at low temperatures. The origin of these localized states is attributed to the formation of Bi-related complexes and to alloy disorder in $\mathrm{Ga}(\mathrm{As}, \mathrm{Bi})$ [4,28-34]. As a matter of fact, our XPS characterization reveals the presence of different Bi configurations in our samples. The extent of the energy redshift is strongly dependent on the growth conditions that can give rise to the formation of different kinds of Bi-related complexes [28,31,33,35,36].

The measured PL peak emission energy of our samples decreases monotonically with increasing Bi concentration [see Fig. 7(c)], similarly to what is expected for the free exciton. Figure 7(b) shows the PL spectra of the three samples T1, T2, and T4, grown under the same conditions as the samples in Fig. 7(a) but on top of the (In, Ga)As DL. Interestingly, despite their slightly lower Bi content, the PL of tensile samples exhibits a further redshift with respect to the corresponding compressive samples, which ranges from 40 to $80 \mathrm{meV}$. This behavior is found to depend on the different hydrostatic strain of the samples grown under compressive and tensile in-plane stresses. Indeed, using the values for $\epsilon_{\|}$and $\epsilon_{\perp}$ of Table I and the GaAs hydrostatic deformation potentials of the conduction and the valence-band edges $a_{c}=-7.17 \mathrm{eV}$ and $a_{v}=-1.16 \mathrm{eV},[16]$ the shift of the band gaps due to the hydrostatic strain, defined as $\delta E_{G}=\left(a_{c}-a_{v}\right)\left(2 \epsilon_{\|}+\epsilon_{\perp}\right)$, is towards higher gaps for the compressive samples (the lattice is globally compressed). On the other hand, it is towards lower gaps for the tensile samples whose lattice is globally expanded. Thus the difference between the band gaps of the compressive and tensile samples having almost the same Bi content, due to the hydrostatic lattice expansion, is $60.52 \mathrm{meV}$ for the C1-T1 couple, $51.93 \mathrm{meV}$ for the C2-T2 one, $75.25 \mathrm{meV}$ for the C4-T4 one, and $56.21 \mathrm{meV}$ for the C4-T5 one, values in good agreement with the experiment. The biaxial strain greatly affects the band gaps, with shifts towards lower gaps for both series of samples, ranging from $200 \mathrm{meV}$ to about $300 \mathrm{meV}$, but since the shifts are in the same direction for both sets of samples, their effects on the band-gap difference between samples under compressive and tensile strains are smaller. Moreover, DFT calculations find a large sensitivity of the band gaps on the $\mathrm{Bi}$ atom arrangement in the GaAs matrix. 
Indeed, the band gap reduces of $107 \mathrm{meV}$ if five $\mathrm{Bi}$ atoms are moved from disperse locations within the 128 atoms unit cell to second-neighbor zinc-blende positions forming a small Ga-Bi cluster. This result confirms that the formation of complexes leads to a further reduction of the alloy band gap.

Our PL results demonstrate that the peak energy of the $\mathrm{Ga}(\mathrm{As}, \mathrm{Bi})$ alloys can be controlled not only by varying the Bi concentration but also by changing the strain conditions, increasing in such a way the degrees of freedom for the tuning of the optical emission. The impact of this result is well represented in Fig. 7(c), where the PL peak energy of the tensile samples is plotted as a function of the Bi concentration and compared to that of the compressive samples and pure GaAs. We can see that, by exploiting a tensile strain, we can shift the energy emissions towards lower values, notably within the range of the telecommunication wavelengths, using less bismuth: an unquestionable advantage given the extreme difficulty of growing highquality material at high Bi concentrations. For example, an emission wavelength of $1.3 \mu \mathrm{m}(0.95 \mathrm{eV})$ can be obtained from $\mathrm{Ga}(\mathrm{As}, \mathrm{Bi})$ grown on $\mathrm{GaAs}$ with a Bi content of $7.5 \%$ while for $\mathrm{Ga}(\mathrm{As}, \mathrm{Bi})$ under tensile strain at a $\mathrm{Bi}$ content of only $4.9 \%$, i.e., the same PL peak energy is achieved with the incorporation of $35 \%$ less $\mathrm{Bi}$, as shown by the dashed black line in Fig. 7(c).

Another important result concerns the PL signal intensity. With the only exception of the couple of samples C1-T1, both containing a very low Bi concentration, the PL efficiency of $\mathrm{Ga}(\mathrm{As}, \mathrm{Bi})$ grown under tensile strain is always higher than that of $\mathrm{Ga}(\mathrm{As}, \mathrm{Bi})$ grown under compressive strain. In the case of the couple C1-T1, the $\mathrm{Ga}(\mathrm{As}, \mathrm{Bi})$ film grown directly on GaAs experiences the lowest mismatch, whereas the sample $\mathrm{T} 1$ is in the opposite situation, i.e., it is under the highest tensile strain. Only in this particular case the growth on an (In, Ga)As DL is not advantageous compared to the growth on a GaAs buffer layer. In Fig. 7(d) the PL intensity is plotted as a function of Bi concentration. Two main regions can be identified: region I for low-intermediate $\mathrm{Bi}$ concentrations $\left[x_{\mathrm{Bi}}<3 \%\right.$, in Fig. 7(d)] and region II for intermediate-high Bi concentrations $\left[x_{\mathrm{Bi}}>4 \%\right.$ in Fig. $\left.7(\mathrm{~d})\right]$. Moving from region I to region II, the PL intensity of the samples under compressive biaxial stress decreases drastically. Conversely, the PL intensity of the samples under tensile biaxial stress preserves the same order of magnitude over a wide range of $\mathrm{Bi}$ concentrations (between 0.74 and $7.2 \%$ ). This is a quite surprising and strongly encouraging result since one would expect a loss of radiative efficiency due to the plastic relaxation of the (In, Ga)As DL with the consequent formation of dislocations and, in general, a high density of nonradiative recombination centers. In the case of samples under compressive strain, it is well known $[27,37]$ that the PL intensity at first increases with increasing Bi content, due to the surfactant effect of $\mathrm{Bi}$, which favors an improvement of the crystal quality, and then decreases at an intermediate Bi content (about 4\%, but strongly dependent on the growth conditions) due to an increase of the density of defects, and an overall worsening of the crystal quality. Thus, at these higher Bi concentrations and related higher compressive biaxial stress, the surfactant action of $\mathrm{Bi}$ seems to be less effective. In the case of $\mathrm{Ga}(\mathrm{As}, \mathrm{Bi})$ under tensile strain, the PL intensity remains high or even increases with increasing Bi contents up to $7.2 \%$. This result suggests that $\mathrm{Ga}(\mathrm{As}, \mathrm{Bi})$ grown under tensile strain exhibits an overall better crystal quality and a reduced defect density. It is worth keeping in mind that for our set of samples under tensile strain an increase of the Bi concentration implies a reduction of the strain, which clearly favors a better crystal growth. However, this cannot be the only explanation. In fact, as shown in Fig. 3, samples $\mathrm{C} 2$ and $\mathrm{C} 3$ have similar absolute strain values as samples T2 and T3, respectively, but the PL intensity of the tensile samples is much higher [Fig. 7(d)]. We speculate that in addition to the lattice expansion caused by the epitaxial tensile strain (we have analyzed previously the effect of the hydrostatic expansion of the volume on the band-gap value), the local strain at the atomic level induced by subsurface stresses is key to the process. It has been demonstrated that surface processes (related to the surface reconstruction and surface stoichiometry) are crucial in determining the final microstructure of $\mathrm{Ga}(\mathrm{As}, \mathrm{Bi})$ layers $[38,39]$. During the growth of tensile samples on the (In, Ga)As DL, Bi atoms impinge on a (In, Ga)As surface characterized by the presence of a bigger atom, In, than $\mathrm{Ga}$ and As atoms of the plain GaAs substrate. As a consequence, the atomic size mismatch between Bi-In-GaAs is significantly reduced compared to Bi-Ga-As, causing a reduction in the local strain at the growth front, which would favor the homogeneous incorporation of $\mathrm{Bi}$ and improve the quality of the layers. In addition, subsurface stresses related to the surface reconstruction in (In, Ga) As DL and the lattice expansion caused by the tensile epitaxial strain may impact adatom mobilities and diffusivities, leading to a more effective Bi surfactant effect and producing crystals with higher quality, as also supported by the Raman characterization (see Appendix B).

\section{SUMMARY}

In order to pursue a strain-engineering strategy on the control of $\mathrm{Bi}$ incorporation in $\mathrm{Ga}(\mathrm{As}, \mathrm{Bi})$ alloy, several films with variable concentration of $\mathrm{Bi}$ are grown by $\mathrm{MBE}$ on bare $\mathrm{GaAs}(001)$ crystals and on partially relaxed $\mathrm{In}_{y} \mathrm{Ga}_{1-y} \mathrm{As} / \mathrm{In}_{z} \mathrm{Ga}_{1-z} \mathrm{As} \quad(y=20 \%-24 \%, z=10 \%-12 \%)$ double layers. Samples are studied from both a crystallographic and structural and optical point of view. Indeed, $\mathrm{Ga}(\mathrm{As}, \mathrm{Bi})$ layers grown on $\mathrm{GaAs}$ are under a compressive strain, whereas the growth on the relaxed (In, Ga)As substrates induces a tensile strain or, for the right $\mathrm{Bi}$ 
concentration, an almost strain-free $\mathrm{Ga}(\mathrm{As}, \mathrm{Bi})$. The different strain conditions strongly impact the $\mathrm{Bi}$ incorporation in the GaAs matrix and the luminescence properties of the sample. In particular, samples grown under tensile strain demonstrate a higher PL efficiency and an interesting higher redshift with respect to the compressive ones. These two effects allowed us to reach the important photoluminescence emission at $1.3 \mu \mathrm{m}$ with a Bi concentration as low as $4.9 \%$ compared to $7.5 \%$ needed for the standard compressive samples. This breakthrough is expected to impact the application of $\mathrm{Ga}(\mathrm{As}, \mathrm{Bi})$ material in optoelectronic devices. The different PL efficiency of the two sets of samples can be traced back to two effects: the different hydrostatic and biaxial strain in the tensile and compressed samples and the improved surfactant effect of $\mathrm{Bi}$ in the tensile samples. In summary, we demonstrate that strain engineering offers a further degree of freedom in the photoluminescence design of $\mathrm{Ga}(\mathrm{As}, \mathrm{Bi})$ material.

\section{ACKNOWLEDGMENTS}

This study is supported by the projects: Consolidate the Foundation 2015 - BILLY - and RSA2017 Mission Sustainability of the University of Rome Tor Vergata. The Bilateral Mobility Program between CNR (SAC.AD002.018.017) (Italy) and the Czech Academy of Sciences (CNR-16-02). Ministry of Education, Youth and Sports of the Czech Republic (Grant No. CZ.02.1.01/0.0/0.0/16_1/0001406).

E.L thanks Sabine Krauß for her dedicated TEM specimen preparation.

Samples were grown and characterized by E.T., E.P., A.Z., and F.A.; XRD measurements were performed by E.T., R.C., and S.Ce. XRD analysis was carried out by S.Ce. AFM characterization was performed by E.T. and E.P. R.M. performed all the theoretical calculations. PL measurements were carried out by E.T., R.F., and P.P. TEM characterization and analysis were performed by E.L. XPS measurements and analysis were performed by J.H., M.V., E.P., S.Co., and F.A. Raman measurements were carried out by E.Z. The paper was written by E.T., R.M., E.P., S.Co, and F.A., with the help and through contributions from all co-authors. All authors have given approval to the final version of the manuscript. The project was initiated and conceptualized by E.P., R.M., and F.A.

\section{APPENDIX A: TEM MEASUREMENTS}

The reason for the chemical sensitivity of $\mathbf{g}_{002}$ DFTEM is that in III-V alloys with ZB structure, the diffracted intensity for the 002 reflection under kinematic approximation is proportional to the square of the structure factor, which in turn depends on the difference in the atomic scattering factors of the alloy components $[22,40]$. Hence, the image contrast in this "structure-factor imaging mode" can be directly correlated with the chemical composition. Due to the different atomic scattering factors involved in the (In, Ga)As and $\mathrm{Ga}(\mathrm{As}, \mathrm{Bi}$ ) layers, (In, Ga)As layers with In concentration $y_{\text {In }} \sim 20 \%$ appear with a very dark contrast, as predicted by theory [40]; while $\mathrm{Ga}(\mathrm{As}, \mathrm{Bi})$ layers exhibit a bright contrast, which increases with increasing Bi content, [24] as clearly observed in Fig. 5.

The quantification of the chemical composition relies on the analysis of the ratio of the intensity diffracted by the layer compared to that of an adjacent reference layer of known composition [22]. In the case of the investigated tensile samples, it would have been fairly simple to use the second (In, Ga)As buffer layer as a reference for the analysis. Unfortunately, the $\mathbf{g}_{002}$ diffracted intensity of (In, Ga)As with In content around $20 \%$ is characterized by a minimum in the intensity [40], giving rise to an extremely dark contrast (cf. Fig. 5) and important uncertainties in the chemical determination. To circumvent this limitation, we develop an alternative strategy where we use the first (In, Ga)As buffer (with $z_{\text {In }} \sim 10 \%$ In) as the reference layer instead. To this end, the procedure requires recording reference $\mathbf{g}_{002}$ DFTEM micrographs of the three layers [Ga(As, Bi), second (In, Ga)As and first (In, Ga)As] simultaneously and under the same imaging conditions. Surface relaxation of the TEM thin foils and local strain fields, in particular at the relaxed (In, Ga)As layers, give rise to local deviations from the exact $\mathbf{g}_{002}$ DFTEM imaging conditions and, hence, impose practical challenges during TEM. Thus, the Bi content estimated in this way is approximate.

\section{APPENDIX B: RAMAN MEASUREMENTS}

The Raman selection rules for the zinc-blende crystal with $T_{d}$ symmetry and the backscattering geometry,

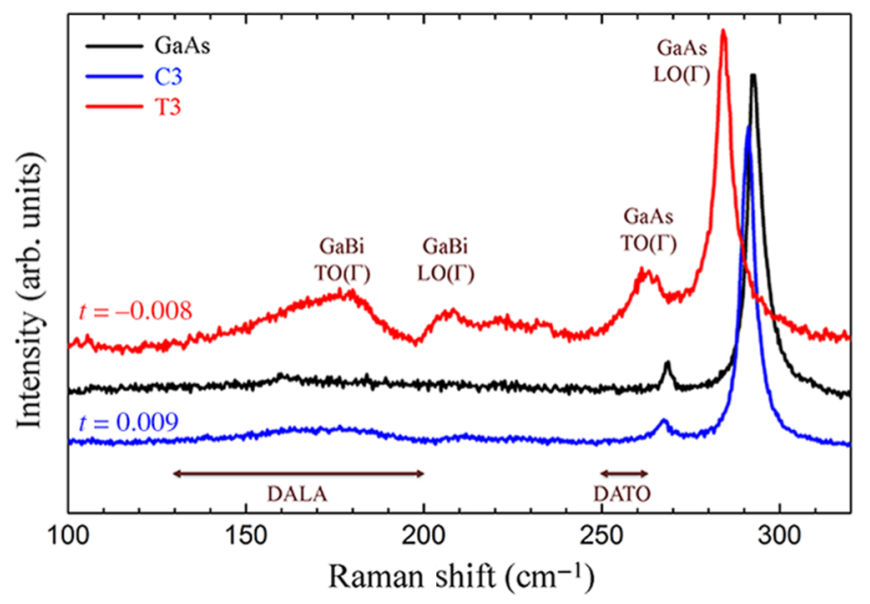

FIG. 8. Raman spectra normalized at $\mathrm{GaAs} \mathrm{LO}(\Gamma)$ peak of $\mathrm{GaAs}$ and $\mathrm{Ga}(\mathrm{As}, \mathrm{Bi})$ samples $\mathrm{C} 3$ and $\mathrm{T} 3$ having a similar absolute value of tetragonal distortion $t$. The different peaks related to Ga-As and Ga-Bi $\mathrm{LO}(\Gamma)$ and $\mathrm{TO}(\Gamma)$ modes are labeled. DATO and DALA modes represent disorder-activated TO modes and disorder-activated longitudinal acoustic modes, respectively. 
allow for the detection of the longitudinal optical phonon $\mathrm{LO}(\Gamma)$, while the transverse optical phonon $\mathrm{TO}(\Gamma)$ is forbidden. The relaxation of the selection rules for the $\mathrm{TO}(\Gamma)$ mode can be attributed to the near-Brewster angle incidence and the large aperture of the collecting lens [41]. For low $\mathrm{Bi}$ concentration the $\mathrm{GaAs} \mathrm{LO}(\Gamma)$ position can be described by the linear relation $[42,43]: \omega_{\mathrm{LO}}\left(\mathrm{cm}^{-1}\right)=$ $\omega_{\mathrm{LO}}^{0}+\Delta \omega_{\mathrm{LO}} x$, where the shift $\Delta \omega_{\mathrm{LO}}$ is related to the strain and the $\mathrm{Bi}$ incorporation by $\Delta \omega_{\mathrm{LO}}=\Delta \omega_{\text {alloy }}+\Delta \omega_{\text {strain }}$.

In order to get further insight into the structural properties of the $\mathrm{Ga}(\mathrm{As}, \mathrm{Bi})$ films, Raman spectroscopy measurements of $\mathrm{GaAs}(001)$ and $\mathrm{C} 3$ and $\mathrm{T} 3 \mathrm{Ga}(\mathrm{As}, \mathrm{Bi})$ samples have been carried out, whose results are reported in Fig. 8. Samples C3 and T3 are chosen because, with a difference in Bi content less than $20 \%$, they have a compressive and tensile tetragonal distortion $t$ of similar magnitude. The spectra show the typical features of bulk GaAs such as the pronounced longitudinal optical phonon (LO) at $292.5 \mathrm{~cm}^{-1}$ and the weaker transverse optical phonon (TO) mode at $268.4 \mathrm{~cm}^{-1}$, both at the high symmetry point $\Gamma$. When $\mathrm{Bi}$ is incorporated substitutional to As, additional Raman modes arise: they are the $\mathrm{GaBi}-\mathrm{LO}(\Gamma)$ and $\mathrm{GaBi}-$ $\mathrm{TO}(\Gamma)$ modes at about 207.5 and $178.9 \mathrm{~cm}^{-1}$, respectively. The intensity of these peaks is directly proportional to the $\mathrm{Bi}$ concentration $[44,45]$. What is important to note in the spectra reported in Fig. 8 is the relative intensity of the GaBi-LO $(\Gamma)$ and $\mathrm{GaBi}-\mathrm{TO}(\Gamma)$ peaks. Despite the small difference in Bi content, these peaks are well defined only for sample T3, while are suppressed in the sample under compressive strain (C3). The intensity and linewidth of these modes depend on the long-range order of the lattice; thus this result should indicate a higher crystal quality of the $\mathrm{Ga}(\mathrm{As}, \mathrm{Bi})$ tensile sample with respect to the compressive one.

From a comparative analysis of the spectra in Fig. 8, we can observe that the energy position of the GaAs- $\mathrm{LO}(\Gamma)$ mode redshifts from $292.5 \mathrm{~cm}^{-1}$ (GaAs) to $284.1 \mathrm{~cm}^{-1}$ (T3), the shift being dependent on Bi concentration and strain [42]. In particular, the compressive sample $\mathrm{C} 3$ shows a smaller redshift of the GaAs- $\mathrm{LO}(\Gamma)$ peak $\left(\Delta \omega_{\mathrm{LO}}=\right.$ $1.2 \mathrm{~cm}^{-1}$ with respect to pure GaAs) than the tensile sample T3 $\left(\Delta \omega_{\mathrm{LO}}=8.4 \mathrm{~cm}^{-1}\right)$ and a very small redshift of the GaAs-TO $(\Gamma)$. The comparison between samples $\mathrm{C} 3$ and T3 evidences the role of the strain. Samples C3 and T3 have $\mathrm{Bi}$ concentrations that differ by less than $20 \%$, while their tetragonal distortion is almost the same but of opposite sign. Therefore, in both cases we expect a redshift of the GaAs- $\mathrm{LO}(\Gamma)$ peak induced by the contribution of $\Delta \omega_{\text {alloy }}$ but an opposite effect of $\Delta \omega_{\text {strain }}$, which induces a redshift for sample T3 and a blueshift for sample C3. The overall effect explains the quite different values of $\Delta \omega_{\mathrm{LO}}$.

As for the GaAs- $\mathrm{TO}(\Gamma)$ peak, its position is dependent on the in-plane lattice parameter, as a matter of fact it is redshifted for the T3 sample [grown on the partially relaxed (In, Ga)As DL] compared to pure GaAs and sample C3, which is pseudomorphic to GaAs. The shoulder at lower energy of $\mathrm{GaAs}-\mathrm{TO}(\Gamma)$ peaks is assigned to disorder-activated TO (DATO) modes. Similarly, disorderactivated longitudinal acoustic (DALA) modes can be observed in the range $150-190 \mathrm{~cm}^{-1}$. These phonons are due to the lattice deformation of the GaAs lattice induced by $\mathrm{Bi}$ incorporation [42] and are more broadened in the $\mathrm{C} 3$ sample.

[1] I. P. Marko, C. A. Broderick, S. Jin, P. Ludewig, W. Stolz, K. Volz, J. M. Rorison, E. P. O'Reilly, and S. J. Sweeney, Optical gain in $\mathrm{GaAsBi} / \mathrm{GaAs}$ quantum well diode lasers, Sci. Rep. 6, 28863 (2016).

[2] R. D. Richards, F. Bastiman, J. S. Roberts, R. Beanland, D. Walker, and J. P. R. David, MBE grown GaAsBi/GaAs multiple quantum well structures: Structural and optical characterization, J. Cryst. Growth 425, 237 (2015).

[3] K. Alberi, O. D. Dubon, W. Walukiewicz, K. M. Yu, K. Bertulis, and A. Krotkus, Valence band anticrossing in $\mathrm{GaBi}_{x} \mathrm{As}_{1-x}$, Appl. Phys. Lett. 91, 051909 (2007).

[4] A. R. Mohmad, F. Bastiman, C. J. Hunter, J. S. Ng, S. J. Sweeney, and J. P. R. David, The effect of Bi composition to the optical quality of $\mathrm{GaAs}_{1-x} \mathrm{Bi}_{x}$, Appl. Phys. Lett. 99, 042107 (2011).

[5] B. Fluegel, S. Francoeur, A. Mascarenhas, S. Tixier, E. C. Young, and T. Tiedje, Giant Spin-Orbit Bowing in $\mathrm{GaAs}_{1-x} \mathrm{Bi}_{x}$, Phys. Rev. Lett. 97, 067205 (2006).

[6] K. K. Nagaraja, Y. A. Mityagin, M. P. Telenkov, and I. P. Kazakov, $\operatorname{Gaas}_{(1-x)} \mathrm{Bi}_{x}$ : A promising material for optoelectronics applications, Crit. Rev. Solid State Mater. Sci. 42, 239 (2017).

[7] S. Tixier, M. Adamcyk, T. Tiedje, S. Francoeur, A. Mascarenhas, P. Wei, and F. Schiettekatte, Molecular beam epitaxy growth of $\mathrm{GaAs}_{1-x} \mathrm{Bi}_{x}$, Appl. Phys. Lett. 82, 2245 (2003).

[8] F. Arciprete and R. Calarco, A special section on effects of strain in semiconductor heterostructures, Nanosci. Nanotechnol. Lett. 9, 1064 (2017).

[9] S. J. Sweeney and J. Mukherjee, Springer Handb. Electron. Photonic Mater. (Springer International Publishing, Cham, 2017), pp. 1-1.

[10] D. Kriegner, E. Wintersberger, and J. Stangl, X-ray utilities: A versatile tool for reciprocal space conversion of scattering data recorded with linear and area detectors, J. Appl. Crystallogr. 46, 1162 (2013).

[11] F. Bastiman, A. G. Cullis, J. P. R. David, and S. J. Sweeney, $\mathrm{Bi}$ incorporation in $\mathrm{GaAs}(100)-2 \times 1$ and $4 \times 3$ reconstructions investigated by RHEED and STM, J. Cryst. Growth 341, 19 (2012)

[12] J. A. Steele, R. A. Lewis, J. Horvat, M. J. B. Nancarrow, M. Henini, D. Fan, Y. I. Mazur, M. Schmidbauer, M. E. Ware, S. Q. Yu, and G. J. Salamo, Surface effects of vapour-liquid-solid driven Bi surface droplets formed during molecular-beam-epitaxy of GaAsBi, Sci. Rep. 6, 1 (2016)

[13] P. Giannozzi et al., QUANTUM ESPRESSO: A modular and open-source software project for quantum simulations 
of materials, J. Phys. Condens. Matter 21, 395502 (2009).

[14] M. B. Giannozzi et al., Advanced capabilities for materials modelling with Q uantum ESPRESSO, J. Phys. Condens. Matter 29, 465901 (2017).

[15] J. P. Perdew and Alex Zunger, Self-interaction correction to density-functional approximations for many-electron systems, Phys. Rev. B 23, 5048 (1981).

[16] I. Vurgaftman, J. R. Meyer, and L. R. Ram-Mohan, Band parameters for III-V compound semiconductors and their alloys, J. Appl. Phys. 89, 5815 (2001).

[17] A. Janotti, Su-Huai Wei, and S. B. Zhang, Theoretical study of the effects of isovalent coalloying of $\mathrm{Bi}$ and $\mathrm{N}$ in GaAs, Phys. Rev. B 65, 115203 (2002).

[18] Heather Jacobsen, Brian Puchala, Thomas F. Kuech, and Dane Morgan, $A b$ initio study of the strain dependent thermodynamics of Bi doping in GaAs, Phys. Rev. B 86, 085207 (2012).

[19] R. Kumar, A. Bag, P. Mukhopadhyay, S. Das, and D. Biswas, Investigation of cross-hatch surface and study of anisotropic relaxation and dislocation on InGaAs on GaAs(001), Electron. Mater. Lett. 12, 356 (2016).

[20] S. Cecchi, E. Gatti, D. Chrastina, J. Frigerio, E. Müller Gubler, D. J. Paul, M. Guzzi, and G. Isella, Thin SiGe virtual substrates for Ge heterostructures integration on silicon, J. Appl. Phys. 115, 093502 (2014).

[21] T. Figielski, A. L. Toth, O. Yastrubchak, T. Wosinski, and E. Lusakowska, Misfit-Dislocation induced surface morphology of InGaAs/GaAs heterostructures, Microchim. Acta 145, 267 (2004).

[22] E. G. Bithell and W. M. Stobbs, Composition determination in the $\mathrm{GaAs} /(\mathrm{Al}, \mathrm{Ga})$ Assystem using contrast in dark-field transmission electron microscope images, Philos. Mag. A 60, 39 (1989).

[23] M. Wu, M. Hanke, E. Luna, J. Puustinen, M. Guina, and A. Trampert, Detecting lateral composition modulation in dilute $\mathrm{Ga}(\mathrm{As}, \mathrm{Bi})$ epilayers, Nanotechnology 26, 425701 (2015).

[24] E. Luna, O. Delorme, L. Cerutti, E. Tournié, J.-B. Rodriguez, and A. Trampert, Microstructure and interface analysis of emerging $\mathrm{Ga}(\mathrm{Sb}, \mathrm{Bi})$ epilayers and $\mathrm{Ga}(\mathrm{Sb}, \mathrm{Bi}) / \mathrm{GaSb}$ quantum wells for optoelectronic applications, Appl. Phys. Lett. 112, 151905 (2018).

[25] J. Honolka, C. Hogan, M. Vondráček, Y. Polyak, F. Arciprete, and E. Placidi, Electronic properties of GaAsBi(001) alloys at low Bi content, Phys. Rev. Mater. 3, 044601 (2019).

[26] P. Laukkanen, M. P. J. Punkkinen, A. Lahti, J. Puustinen, M. Tuominen, J. Hilska, J. Mäkelä, J. Dahl, M. Yasir, M. Kuzmin, J. R. Osiecki, K. Schulte, M. Guina, and $\mathrm{K}$. Kokko, Local variation in $\mathrm{Bi}$ crystal sites of epitaxial GaAsBi studied by photoelectron spectroscopy and first-principles calculations, Appl. Surf. Sci. 396, 688 (2017).

[27] X. Lu, D. A. Beaton, R. B. Lewis, T. Tiedje, and Y. Zhang, Composition dependence of photoluminescence of $\mathrm{GaAs}_{1-x} \mathrm{Bi}_{x}$ alloys, Appl. Phys. Lett. 95, 041903 (2009).

[28] Muhammad Usman, Christofer A. Broderick, Andrew Lindsay, and Eoin P. O'Reilly, Tight-binding analysis of the electronic structure of dilute bismide alloys of $\mathrm{GaP}$ and GaAs, Phys. Rev. B 84, 245202 (2011).

[29] S. Mazzucato, H. Lehec, H. Carrère, H. Makhloufi, A. Arnoult, C. Fontaine, T. Amand, and X. Marie, Low-temperature photoluminescence study of exciton recombination in bulk GaAsbi, Nanoscale Res. Lett. 9, 19 (2014).

[30] F. Sarcan, Ö Dönmez, K. Kara, A. Erol, E. Akalın, M. Çetin Arıkan, H. Makhloufi, A. Arnoult, and C. Fontaine, Bismuth-induced effects on optical, lattice vibrational, and structural properties of bulk GaAsBi alloys, Nanoscale Res. Lett. 9, 119 (2014).

[31] T. Wilson, N. P. Hylton, Y. Harada, P. Pearce, D. AlonsoÁlvarez, A. Mellor, R. D. Richards, J. P. R. David, and N. J. Ekins-Daukes, Assessing the nature of the distribution of localised states in bulk GaAsBi, Sci. Rep. 8, 6457 (2018).

[32] S. Francoeur, S. Tixier, E. Young, T. Tiedje, and A. Mascarenhas, Bi isoelectronic impurities in GaAs, Phys. Rev. B 77, 085209 (2008)

[33] G. Ciatto, E. C. Young, F. Glas, J. Chen, R. Alonzo Mori, and $\mathrm{T}$. Tiedje, Spatial correlation between $\mathrm{Bi}$ atoms in dilute $\mathrm{GaAs}_{1-x} \mathrm{Bi}_{x}$ : From random distribution to $\mathrm{Bi}$ pairing and clustering, Phys. Rev. B 78, 035325 (2008).

[34] B. Yan, X. Chen, L. Zhu, W. Pan, L. Wang, L. Yue, X. Zhang, L. Han, F. Liu, S. Wang, and J. Shao, Bismuthinduced band-tail states in GaAsBi probed by photoluminescence, Appl. Phys. Lett. 114, 052104 (2019).

[35] T. M. Christian, K. Alberi, D. A. Beaton, and B. Fluegel, Spectrally resolved localized states in $\mathrm{GaAs}_{1-x} \mathrm{Bi}_{x}$, Jpn. J. Appl. Phys. 56, 035801 (2017).

[36] K. Alberi, T. M. Christian, B. Fluegel, S. A. Crooker, D. A. Beaton, and A. Mascarenhas, Localization behavior at bound $\mathrm{Bi}$ complex states in $\mathrm{GaAs}_{1-x} \mathrm{Bi}_{x}$, Phys. Rev. Mater. 1, 024605 (2017).

[37] R. D. Richards, F. Bastiman, C. J. Hunter, D. F. Mendes, A. R. Mohmad, J. S. Roberts, and J. P. R. David, Molecular beam epitaxy growth of GaAsBi using $\mathrm{As}_{2}$ and $\mathrm{As}_{4}, \mathrm{~J}$. Cryst. Growth 390, 120 (2014).

[38] E. Luna, M. Wu, J. Puustinen, M. Guina, and A. Trampert, Spontaneous formation of nanostructures by surface spinodal decomposition in $\mathrm{GaAs}_{1-x} \mathrm{Bi}_{x}$ epilayers, J. Appl. Phys. 117, 185302 (2015).

[39] E. Luna, M. Wu, T. Aoki, M. R. McCartney, J. Puustinen, J. Hilska, M. Guina, D. J. Smith, and A. Trampert, Impact of $\mathrm{Bi}$ incorporation on the evolution of microstructure during growth of low-temperature $\mathrm{GaAs}: \mathrm{Bi} / \mathrm{Ga}(\mathrm{As}, \mathrm{Bi})$ layers, J. Appl. Phys. 126, 085305 (2019).

[40] H. Cerva, Transmission electron microscopy of heteroepitaxial layer structures, Appl. Surf. Sci. 50, 19 (1991).

[41] Z. P. Wang, H. X. Han, G. H. Li, D. S. Jiang, and K. Ploog, Raman scattering from to phonons in $(\mathrm{GaAs})_{\mathrm{n}} /(\mathrm{AlAs})_{\mathrm{n}}$ superlattices, Phys. Rev. B 38, 8483 (1988).

[42] J. A. Steele, R. A. Lewis, M. Henini, O. M. Lemine, and A. Alkaoud, Raman scattering studies of strain effects in (100) and (311)B $\mathrm{GaAs}_{1-x} \mathrm{Bi}_{x}$ epitaxial layers, J. Appl. Phys. 114, 193516 (2013).

[43] P. Wang, W. Pan, X. Wu, C. Cao, S. Wang, and Q. Gong, Heteroepitaxy growth of GaAsBi on $\mathrm{Ge}(100)$ substrate by 
gas source molecular beam epitaxy, Appl. Phys. Express 9, 045502 (2016).

[44] J. A. Steele, R. A. Lewis, M. Henini, O. M. Lemine, D. Fan, Y. I. Mazur, V. G. Dorogan, P. C. Grant, S.-Q. Yu, and G. J. Salamo, Raman scattering reveals strong LO-phonon-hole-plasmon coupling in nominally undoped GaAsBi: Optical determination of carrier concentration, Opt. Express 22, 11680 (2014).

[45] P. Verma, K. Oe, M. Yamada, H. Harima, M. Herms, and G. Irmer, Raman studies on $\mathrm{GaAs}_{1-x} \mathrm{Bi}_{x}$ and $\operatorname{InAs}_{1-x} \mathrm{Bi}_{x}$, J. Appl. Phys. 89, 1657 (2001). 